OPINION EDITORIAL

\title{
S.H.A.K.E. COVID-19
}

EXERCISE IS MEDICINE

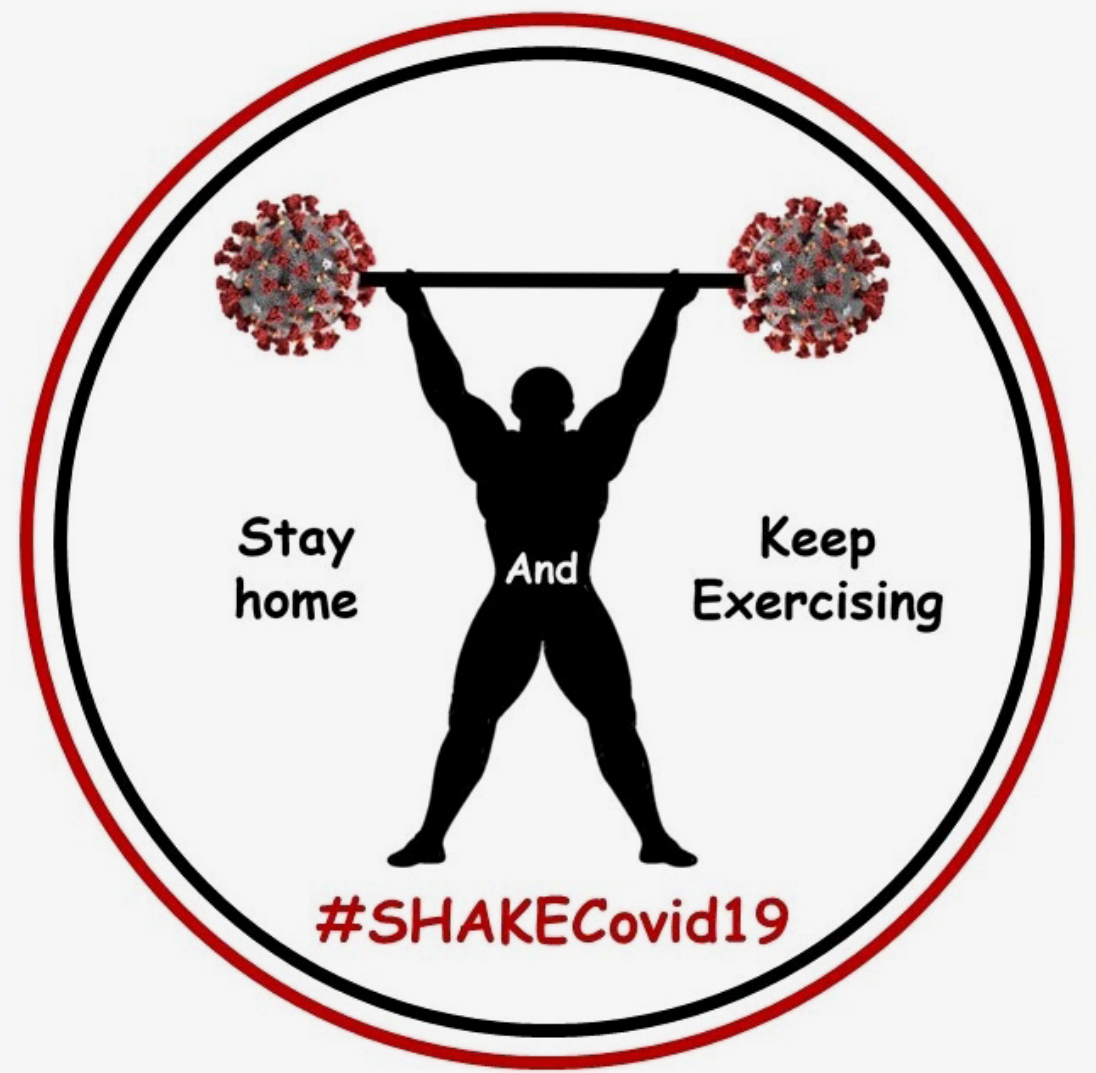

\section{Stay Home And Keep Exercising}

Mitrousias Vasileios, Kyriaki Baxevanidou, Zibis Aristeidis

Department of Anatomy, Faculty of Medicine, University of Thessaly, Larissa, Greece

It's been a while since Coronavirus disease (COVID-19) was characterized as a pandemic by the World Health Organization (WHO), causing major concerns and unfortunately panic in many countries. The ongoing outbreak is of international concern and most countries have already announced a series of protective measures to confront this health threat of unknown magnitude. Travel warnings, scientific congress suspensions, cancellations of national holidays and closing of schools, amusement parks, cinemas, theatres and gyms are only some of these measures.

The impact in sports is also tremendous. Major sport events like the 2020 Tokyo Olympic Games and Euro 
2020 have been postponed. Others like the Miami Open and the Wimbledon tennis tournament have been cancelled and there is an ongoing discussion for forthcoming events. However, few have been said regarding the impact of limiting public exercise.

There are hundreds of research papers allowing for rapid sharing of information about the virus the last 20 days, however many questions about transmission, incubation period and treatment remain to be answered. To address this urgent public health threat, avoiding human-to-human transmission of the virus by staying at home is one of the most common directives, limiting the daily outdoor activities of millions of people. Inevitably, staying at home is one of the few effective measures, if not the only one, but it is also this measure that may create a new challenge.

It is likely that staying at home for prolonged time may increase sedentary habits like sitting on the couch, watching tv or playing video games. It is also possible, that in these hard times, people will search for "junk food happiness". Undoubtably, if "stay at home" warnings continue, we will be soon facing a new challenge, a new danger, an increased risk for and potential worsening of health conditions, including the chronic ones.

Exercise is both prevention and treatment. Its effect on many diseases like diabetes or heart coronary disease is undoubtable [1]. Its importance in lowering the risk of many types of cancers is proven [2]. It also boosts immunity and can reduce the risk of falling prey to upper respiratory tract infections [3]. What more could one ask for? Eating healthy is equally important, sharing or enhancing exercise's effects [4]. And it is a physician's responsibility to promote physical activity and healthy eating habits [5]. Exercise prescription is not the next big thing. It is a demanding reality, a haste to address the new challenges and preserve patients' adherence rates to physical activity interventions.

Exercise at home using various, common but safe, equipment is of great importance to maintain adequate fitness levels and fight not only - but also COVID-19. Exercises, either aerobic or resistance, should be prescribed to patients but also healthy individuals. Even the simpler ones could be used. Stationary bicycle, walking to carry the necessary groceries, stair stepping, push-ups, jump rope, tv-instructed pilates, water bottle weights or even dancing in the living room, all could be beneficial under the current difficult circumstances. Anyway, current literature is full of home-based exercise programs. However, it is the physicians' duty to remind to the public that even a minimum of 150 minutes of weekly exercise is effective.

Current directives recommend staying at home. "For how long?" is still under question. It is our responsibility to keep ourselves and our patients healthy. For all physicians, regardless of their specialty, it is not just "stay at home", it is "stay active and stay healthy at home"! And since we are living in the era of social media motivation, use the slogan \#SHAKECovid19. Stay Home And Keep Exercising, to fight the COVID-19 outbreak. Let us share a new directive!

\section{Acknowledgements}

None.

\section{Competing interests}

Nothing to declare. 


\section{Funding info}

No funding to declare.

\section{Ethical approval information}

There was no need for ethical approval for the current manuscript.

\section{Corresponding author}

Vasileios Mitrousias, MD, PhD

University of Thessaly

Faculty of Medicine

Department of Anatomy

Panepistimion 3st Biopolis

41110, Larissa, Greece

tel. +306979051248

fax. +302410289266

E-mail: vasileiosmitrousias@gmail.com

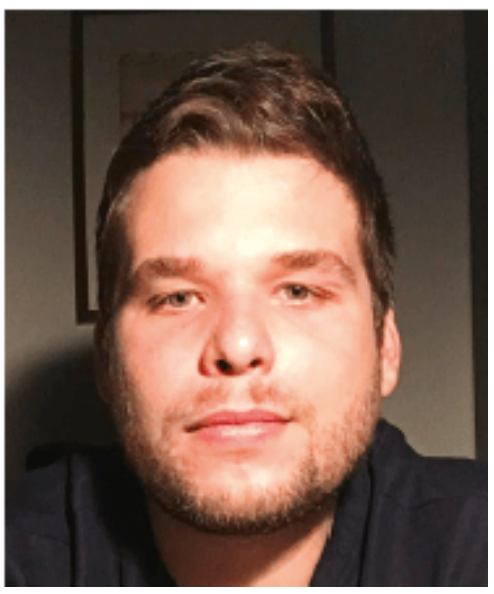

\section{References}

1. Anderson E, Durstine JL. Physical activity, exercise, and chronic diseases: A brief review. Sports Medicine and Health Science 2019;1:3-10. https://doi.org/10.1016/j.smhs.2019.08.006.

2. Friedenreich CM, Stone CR, Cheung WY, Hayes SC. Physical Activity and Mortality in Cancer Survivors: A Systematic Review and Meta-Analysis. JNCI Cancer Spectrum 2020;4. https://doi.org/10.1093/JNCICS/PKZ080.

3. Nieman DC, Wentz LM. The compelling link between physical activity and the body's defense system. Journal of Sport and Health Science 2019;8:201-17. https://doi.org/10.1016/j.jshs.2018.09.009.

4. Schulze MB, Martinez-Gonzalez MA, Fung TT, Lichtenstein AH, Forouhi NG. Food based dietary patterns and chronic disease prevention. BMJ (Clinical Research Ed) 2018;361:k2396. https://doi.org/ 10.1136/bmj.k2396.

5. AOMRC. Exercise: The miracle cure and the role of the doctor in promoting it. 2015. https://www.aomrc.org.uk/wp-content/uploads/2016/05/Exercise_the_Miracle_Cure_0215.pdf (accessed March 17, 2020). 